\title{
Handheld Digital TV Performance Evaluation Method
}

\author{
Gunnar Bedicks Jr., Fujio Yamada, Francisco Sukys, Cristiano Akamine, \\ Luis Tadeu Raunheitte, and Edson L. Horta
}

Mackenzie Digital Television Laboratory, Mackenzie Presbyterian University, Rua da Consolacao 930, Sao Paulo, CEP 01302-907, SP, Brazil

Correspondence should be addressed to Fujio Yamada, fyamada@mackenzie.com.br

Received 17 December 2007; Revised 8 June 2008; Accepted 17 September 2008

Recommended by Jörn Altmann

This paper presents a simplified method for signal level clearance evaluation in each place of signal reception up to threshold for a given portable TV receiver device. It describes briefly the structure of transmission system considering the "1Seg" structure of ISDB-T. The preliminary laboratory test was made before the field tests to verify the main features of handheld receiver.

Copyright ( $) 2008$ Gunnar Bedicks Jr. et al. This is an open access article distributed under the Creative Commons Attribution License, which permits unrestricted use, distribution, and reproduction in any medium, provided the original work is properly cited.

\section{INTRODUCTION}

The new generation of handheld devices predicts that the consumption and use of mobile and portable television sets will change our habits as the use of cellular phones revolutionized the telecommunication business. People enjoy having in the video receiver information about news, sports, weather, traffic conditions and shows at anytime and anyplace without any access restrictions. The handheld device manufacturer desires to evaluate the performance of the product, for each environment, before sending it to the market. Usually, handheld devices circuits are compact and have no access inside the circuitry to connect measurement instruments. This work presents a simplified method to evaluate the device performance data and the level clearance (how much the signal would degrade and still have a good reception). This method can be useful for broadcaster to test the performance of its system and the portable receiver.

The main transmission systems possibilities to provide live television services on handheld devices currently available are as follows: T-DMB, S-DMB, Media FLO, DVB-H, and ISDB-T, all of them using OFDM modulation.

Digital video broadcasting handheld (DVB-H) uses a time-sliced transmission scheme allowing turn the receiver circuit off, for those time slices that are not of interest to the user and thus reducing the power consumption used for TV reception. A significant advanced feature is the
H.264/AVC coding providing efficient coding. The optional multiprotocol encapsulation (MPE)FEC offers a solid error protection $[1,2]$.

Media following link only (Media FLO) is able to dispose 15 to 20 channels of real time broadcast TV displayed on a mobile phone at 30 frames per second scanning in HD resolutions. It uses hierarchical modulation supporting multiple channels of live contents and downloaded clips and forward error corrector techniques. It uses H.264 video coding and audio AAC coding [3, 4].

Digital multimedia broadcast (T-DMB) delivers mobile television services based on the Eureka-147 digital audio broadcast (DAB) standard with additional error correction code offering a capability of 12 to $15 \mathrm{Mbps}$, using the bandwidth of $1.536 \mathrm{MHz}$ for mobile digital TV reception [5].

Integrated services digital broadcast terrestrial (ISDB$\mathrm{T}$ ) provides one mode suitable to broadcast to handheld devices named "1Seg" transmission. The $6 \mathrm{MHz}$ channel band is divided into 13 segments of $430 \mathrm{kHz}$ bandwidth each and one of them can be suited for portable digital TV reception. This method is known as band-segmented transmission OFDM (BST-OFDM) in same way of DVB$\mathrm{H}$ system. For one segment, it is used transmission H.264 encoder, providing a higher digital compression rate than MPEG-2. Also, the QPSK modulation scheme associated to OFDM allows strong robustness against the multipath, burst noise and Doppler effect even in low $C / N$. ISDB-T allows 
up to three simultaneous transmission layers in the same bandwidth. As well, a modulation method with different robustness degree can be selected for each layer [6, 7].

This paper describes the procedure for evaluation the clearance that exists in each place of digital signal reception. A field trial was carried out in São Paulo to determine the viability of the proposed method using mobile devices with "1Seg" ISDB-T transmission. A $1 \mathrm{~kW}$ transmitter tuned on channel $24(530 \sim 536 \mathrm{MHz})$ was installed with a 100 meter height tower. The modulation scheme was configured to have twelve segments for HDTV transmission and one segment for broadcasting to handheld receivers. Several reception sites distributed in main regions of the city were selected to evaluate the reception signal quality.

\section{BACKGROUND}

The band-segmented transmission (BST)-OFDM modulation scheme used in ISDB-T system was developed to broadcast digital terrestrial TV with flexible modulation. It is something similar to hierarchical transmission used in DVB$\mathrm{H}$. The main difference is that in BST-OFDM, the $6 \mathrm{MHz}$ channel bandwidth is divided into 13 segments, grouped in up to three hierarchical levels, with different modulation configuration and robustness. Brazil adopted the system ISDB-T modified, using H264 code instead of MPEG-2. With this structure, it is possible to transmit one HDTV signal with 64-QAM scheme, one program for vehicular reception in 16QAM, and one segment in QPSK. It was used in this test the 1Seg transmission structure modulated in QPSK together with a HDTV transmission using 12 segments.

The OFDM concept is based on spreading the data to be transmitted over a large number of carriers, each being modulated at a low bit rate. There is no interference between carriers, even with a degree of spectral overlap because the carrier spacing is selected so they are orthogonal over the symbol period, and thus, the symbol can be recovered without degradation [7].

For maximum spectral efficiency, the carrier spacing is equal to the reciprocal of the symbol period. The multiplex of the carrier may be conveniently generated digitally using the inverse fast Fourier transformer (IFFT) process.

The carrier spacing in OFDM system is inversely proportional to the symbol length to achieve the orthogonality, for this reason, the number of carriers in a channel is determined from the symbol length. In order to obtain reasonable useful bitrate, a guard interval is used to remove the effect of intersymbolic interference.

\section{PRELIMINARY LABORATORY TEST}

The aim of this step was to verify the performance of the handheld devices sold in regular commerce. For the test, it was used an ISDB-T one segment receiver. These types of apparatus have no available facilities to access inside circuits to be measured. So, it was necessary to develop an expedient to get these data without changing the receiver characteristics. The alternative was to fasten the handheld receiver antenna close to a spectrum analyzer antenna used

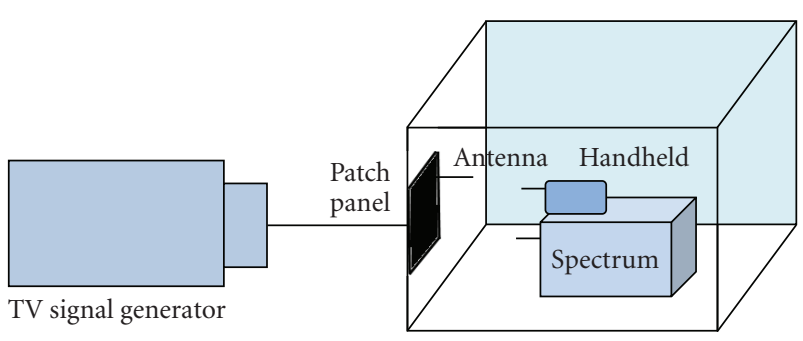

Faraday cage

FIGURE 1: Laboratory installation.

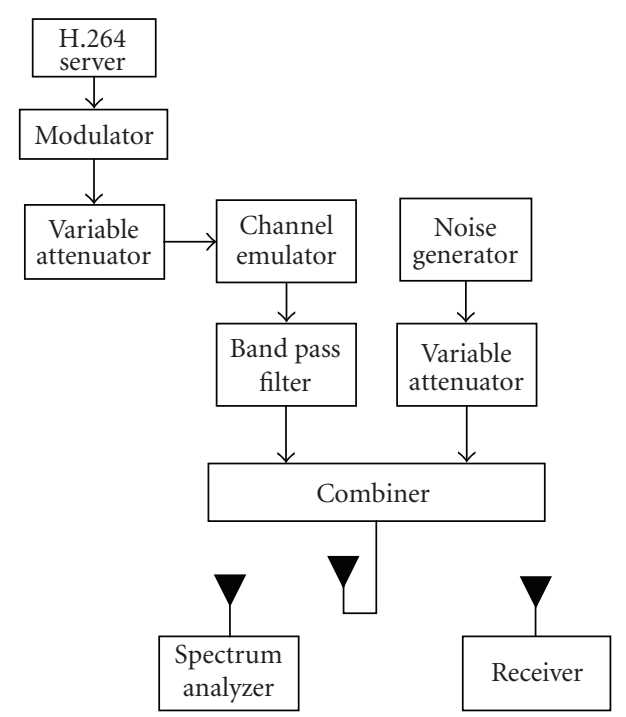

FIgURE 2: Handheld feature measurement setup.

to measure the field strength of the TV channel signal searched (Figure 1). For this purpose, the antenna installed in the spectrum analyzer was constructed to be similar to that used in the handheld device, considering that both antennas were capturing just the same signal strength. To avoid nondesired disturbance, the handheld receiver and the spectrum analyzer were installed inside a Faraday cage.

The transmission signal was generated by a broadcast tester generator modulated by one HDTV signal using 12 segments and a handheld signal using only one segment with H264 coder and configured in a strong scheme as QPSK constellation, guard interval 1/16, FEC 1/2, temporal interleaver 0, 4 seconds, and $330 \mathrm{Kbps}$ bitrate. This signal was radiated inside the Faraday cage by an antenna free of the external disturbance.

Figure 2 summarizes the handheld features measurement setup. The spectrum analyzer and receiver antennas, assembled together in parallel, pick up same field intensity level. The attenuators allow adjusting the level for desired value. The following main features were measured.

\section{(i) Minimum signal level}

The attenuator was adjusted to have a level of about $-40 \mathrm{dbm}$ measured by spectrum analyzer within the "one segment 
TABLE 1: Feature of handheld sample.

\begin{tabular}{lcc}
\hline Feature & HDTV & Handheld \\
\hline Minimum signal level & $-71.3 \mathrm{dBm}$ & $-82.4 \mathrm{dBm}$ \\
$C / N$ (Carrier to Noise) & $18 \mathrm{~dB}$ & $2.7 \mathrm{~dB}$ \\
Multipath (Brazil D) & $11.6 \mathrm{~dB}$ & $11.6 \mathrm{~dB}$ \\
\hline
\end{tabular}

band" $(430 \mathrm{kHz})$. Gradually, the signal level was reduced until the image freezes, reaching the limit of perceptibility (LOP). Table 1 shows the results obtained.

\section{(ii) Carrier to noise ratio at the limit of perceptibility (LOP)}

The signal level $(C)$ was adjusted to about $-40 \mathrm{dBm}$. After that, it was injected additional Gaussian noise $(N)$ up to the image locks. Table 1 indicates the calculated $C / N$ value in $\mathrm{dB}$

\section{(iii) Multipath interference}

It was injected a signal generated by channel emulator configured to reproduce the "Brazil D" multipath spectrum, composed by 5 signals delayed from the main signal. Note that "Brazil D" represents a typical indoor reception configuration. The carrier signal level $(C)$ was adjusted to $-40 \mathrm{dBm}$ and then it was injected additional Gaussian noise $(N)$ until the image reached the LOP. The $C / N$ calculated value is presented in Table 1.

\section{FIELD TEST PROCEDURES}

The aim of this test was to evaluate in the field the behavior of a handheld device using a simplified procedure and, at the same time, to verify the signal level clearance available for this receiver. The results presented here are only samples of the test procedure, but not necessarily a conclusion. For this test, it was used a one segment handheld TV receiver sold in Japan commerce.

As the users of mobile devices are in movement, the signal arriving in the apparatus depends on conditions, like place where they are at each moment, existence of buildings, presence of the people, the direction that the device antenna is pointed to, and the height of the device antenna. Besides, because the researcher needs to move between crowds, the measurement setup must be simple and flexible allowing easy transport. So, taking simplicity as a goal, the equipment suited for use at the field was a portable spectrum analyzer aggregated to the handheld receiver as shown in Figure 3.

As the field strength for mobile devices is very instable; the measurements must be repeated in the same place, varying the antenna point out direction to evaluate the average value. The number of selected test points must represent the morphology of a large city in real signal propagation conditions.

Figure 4 shows the transmission test setup broadcasting one HDTV program with 12 segments and one program for handheld receiver using one segment, in the same $6 \mathrm{MHz}$ channel. The modulation for "one segment" was QPSK. It was used an $1 \mathrm{~kW}$ transmitter with omni antenna, tuned

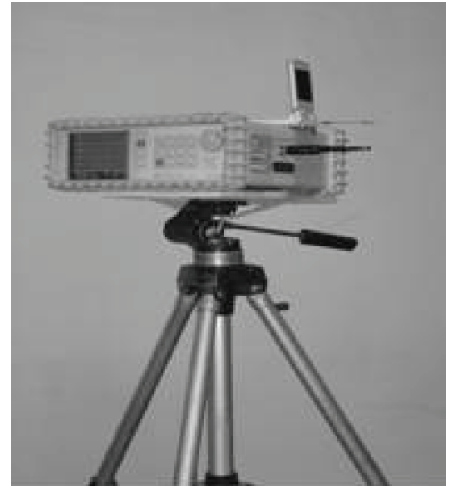

FIgURE 3: Field test setup.

to channel $24(533 \mathrm{MHz})$. A band pass filter was used to restrict the noise to a band near the value of the "one segment TV" band, necessary to not overload the transmitter power amplifier.

In summary, the field test procedures to evaluate the signal clearance are as follows.

(i) Adjust the transmitter to radiate one segment program.

(ii) Measure the signal level $C(\mathrm{dBm})$ for one segment band in the transmitter side (point B-Figure 4).

(iii) Install the handheld receiver together with the spectrum analyzer in each selected site. Measure the received signal level, $C_{1}(\mathrm{dBm})$.

(iv) In the transmitter side, inject gradually the Gaussian noise varying the noise attenuator up to the receiver locks. Read the value of the injected noise $N_{j(\mathrm{Tx})}$ in $\mathrm{dBm}$ (point C-Figure 4).

(v) Repeat the above procedures for other measurements at same site, changing the antenna directions, rotating of $90^{\circ}$ each time [8] to found the signal average value.

\subsection{Signal clearance calculation procedure}

\subsubsection{Definition}

The clearance is defined as

$$
10 \log \left(\frac{N}{N_{a}}\right) \mathrm{dB},
$$

where $N=$ noise power within the one segment band $(430 \mathrm{kHz})$ that makes the receiver reach the limit of perceptibility (LOP), $N_{a}=$ interfering noise power at the receiver input in one segment band $(430 \mathrm{kHz})$.

\subsubsection{Signal clearance calculation}

(i) As measured in laboratory, the LOP reference noise in the receiver for channel configuration used is $2.7 \mathrm{~dB}$ below the signal level input $C_{1}(\mathrm{dBm})$ (see Table 1). So,

$$
N_{(\mathrm{dBm})}=C_{(\mathrm{dB})}-X_{(\mathrm{dB})},
$$

where $X_{(\mathrm{dB})}=C / N=2.7 \mathrm{~dB}($ from Table 1$)$. 


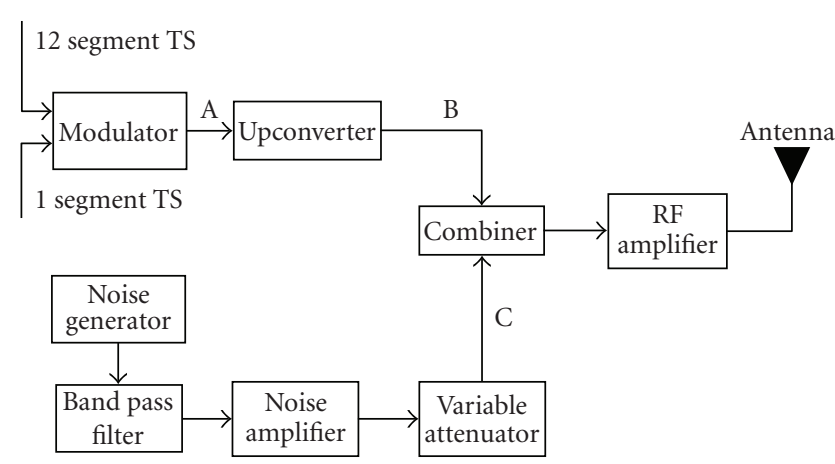

FIgURE 4: Transmission setup.

(ii) When the receiver reaches LOP

$$
N=N_{a}+N_{j} \quad \text { or } \quad N_{a}=N-N_{j},
$$

where $N_{j}=$ equivalent noise injected, at the receiver side, over the interfering noise power $N_{a}$

$$
N_{j}=\frac{N_{j(\mathrm{Tx})}}{k},
$$

where $N_{j(\mathrm{Tx})}$ was converted from $N_{j(\mathrm{Tx})} \mathrm{dBm}$ into power unit, $k=$ attenuation factor and may be calculated as

$$
k=\frac{C_{(\mathrm{Tx})}}{C_{1}},
$$

where $C_{(\mathrm{Tx})}=$ power injected into the one segment band at the transmitter side (measured) converted from $C_{(\mathrm{Tx})} \mathrm{dBm}$; $C_{1}=$ input power at the receiver device.

(iii) By using clearance definition

$$
M_{\mathrm{dB}}=10 \log \left(\frac{N}{N_{a}}\right)
$$

from (3)

$$
N_{a}=N-N_{j},
$$

and from (4)

$$
N_{j}=\frac{N_{j(\mathrm{Tx})}}{k},
$$

so,

$$
M_{\mathrm{dB}}=10 \log \left(\frac{N}{N-N_{j(\mathrm{Tx})} / k}\right)
$$

where $k$ is obtained from (5).

The transmitter signal level at the point B (see Figure 4) was fixed at $-27 \mathrm{dBm}$. The variable Gaussian noise $N_{j(\mathrm{Tx})}$ injected at the point $\mathrm{C}$ (see Figure 4 ) is exhibited in the Table 2.

With the data collected in the field, the level clearance

\begin{tabular}{|c|c|c|c|c|c|}
\hline $\begin{array}{l}\text { Point } N^{\circ} \\
*\end{array}$ & $\begin{array}{l}\text { Dist } \\
\mathrm{Km}\end{array}$ & $\begin{array}{l}N_{j(\mathrm{Tx})} \\
\mathrm{dBm}\end{array}$ & $\begin{array}{c}\text { Average Signal } C_{1} \\
\text { dBm }\end{array}$ & $\begin{array}{c}\text { Max. Signal } \\
\text { dBm }\end{array}$ & $\begin{array}{c}\text { Clearance } \\
\mathrm{dB}\end{array}$ \\
\hline 1 & 2 & -30.91 & -75.7 & -73.1 & 5.8 \\
\hline 2 & 2 & -30.11 & -72.5 & -71.1 & 9.6 \\
\hline 3 & 2 & -30.62 & -77.9 & -76.1 & 6.8 \\
\hline 4 & 2 & -30.33 & -63.5 & -61.1 & 8.1 \\
\hline 5 & 2 & -30.43 & -52.5 & -51.1 & 7.6 \\
\hline 6 & 2 & -30.71 & -71.1 & -69.1 & 6.5 \\
\hline 7 & 5 & -30.57 & -52.2 & -51.0 & 7.0 \\
\hline 8 & 5 & -30.81 & -81.3 & -81.6 & 6.1 \\
\hline 9 & 5 & -29.81 & -73.4 & -71.0 & 13.3 \\
\hline 10 & 5 & -29.81 & -65.7 & -64.0 & 13.3 \\
\hline 11 & 10 & -30.43 & -88.0 & -88.0 & 7.6 \\
\hline
\end{tabular}
was calculated according to the procedure described above. The clearance value means what is the signal level tolerance, existing in each site to keep the reception in a good condition. This clearance level depends on the signal level and the noise level that exist in each point.
TABLE 2: Field measurement results.

\subsection{Field measurement samples}

The samples data of measurements carried out in São Paulo city for sites located at $2 \mathrm{~km}, 5 \mathrm{~km}$, and $10 \mathrm{~km}$ from the transmitter allows to calculate the field intensity and the signal level clearance still existing in each site measured as shown in Table 2. The above distances were selected due to the maximum transmitter power authorized for test by Brazilian Communications Regulatory Agency was only $1 \mathrm{~kW}$.

\section{CONCLUSIONS}

Today, there are many products for mobile handheld reception available in commerce and this fact opened the possibility to Mackenzie Presbyterian University to carry out a field test of these apparatus types by simplified method. The aim of this work was to develop a simple and reliable field test procedure allowing to easily executing the outdoor and indoor tests since the unique instruments necessary to do it are a spectrum analyzer with the attached receiver and a cellular phone to communicate with the transmitter site. The field tests were done and they confirmed the proposed procedures are achievable. The field tests also attested the reception robustness and quality of the handheld devices used, presenting promising results for this type of broadcasting.

\section{ACKNOWLEDGMENTS}

This work was supported with the funds from Mackpesquisa. The authors Fujio Yamada, Gunnar Bedicks Jr., Francisco Sukys, Cristiano Akamine, Luis Tadeu Raunheitte, and Edson L. Horta from Mackenzie Presbyterian University, São Paulo, Brazil are working on digital TV research. They were on charge for suggesting the transmission and reception, Channel coding and modulation of Brazilian digital television system are now being to install in Brazil. 


\section{REFERENCES}

[1] ETSI EN DVB-H SYS Digital Video Broadcasting, "Transmission to Handheld Terminal DVB-H Implementation Guide Line".

[2] G. Faria, J. A. Henriksson, E. Stare, and P. Talmola, "DVB-H: digital broadcast services to handheld devices," Proceedings of the IEEE, vol. 94, no. 1, pp. 194-209, 2006.

[3] Media FLO Technology Overview, Qualccom.

[4] M. R. Chari, F. Ling, A. Mantravadi, et al., "FLO physical layer: an overview," IEEE Transactions on Broadcasting, vol. 53, no. 1, pp. 145-159, 2007.

[5] ETSI TS 102428 V.1.1, DAB, DMB Video Service, User Applications Specifications. 2006.

[6] S. Miyake, "Digital Terrestrial Broadcasting Mobile Environment," NHK Science and Technical Research Laboratories, April 2003.

[7] Y. Takahashi and Y. Dewa, "Outline of digital broadcasting in Japan, outline of ISDB-T system," in ISDB-T Seminar in Brazil, São Paulo, Brazil, March 2005.

[8] F. Yamada, G. Bedicks, F. Sukys, L. T. M. Raunheitte, and C. Akamine, "Sistema de TV digital," in Procedimento de Teste, Revista Mackenzie de Engenharia e Computação Ano 5, vol. 5, São Paulo, Brazil, 2005. 

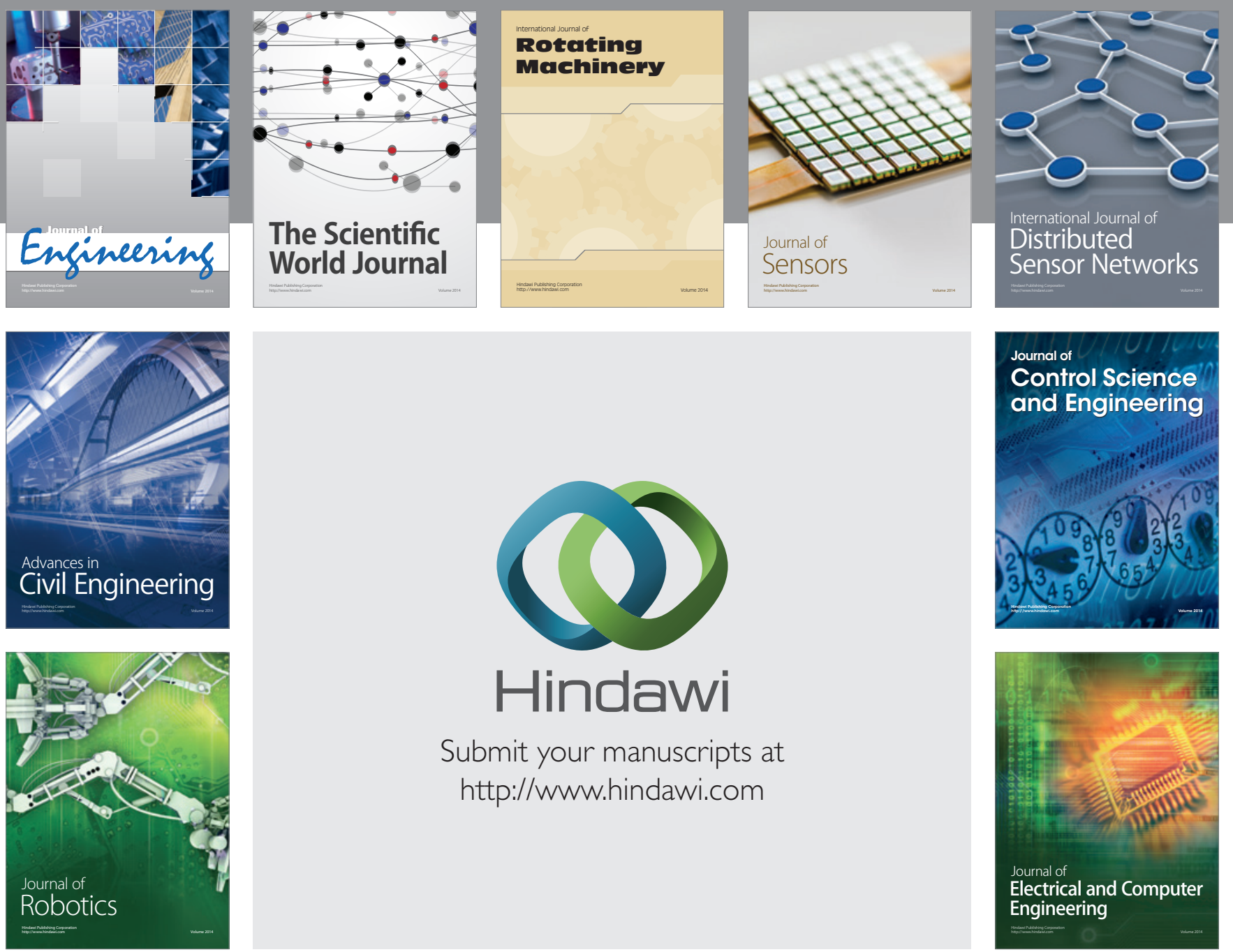

Submit your manuscripts at

http://www.hindawi.com
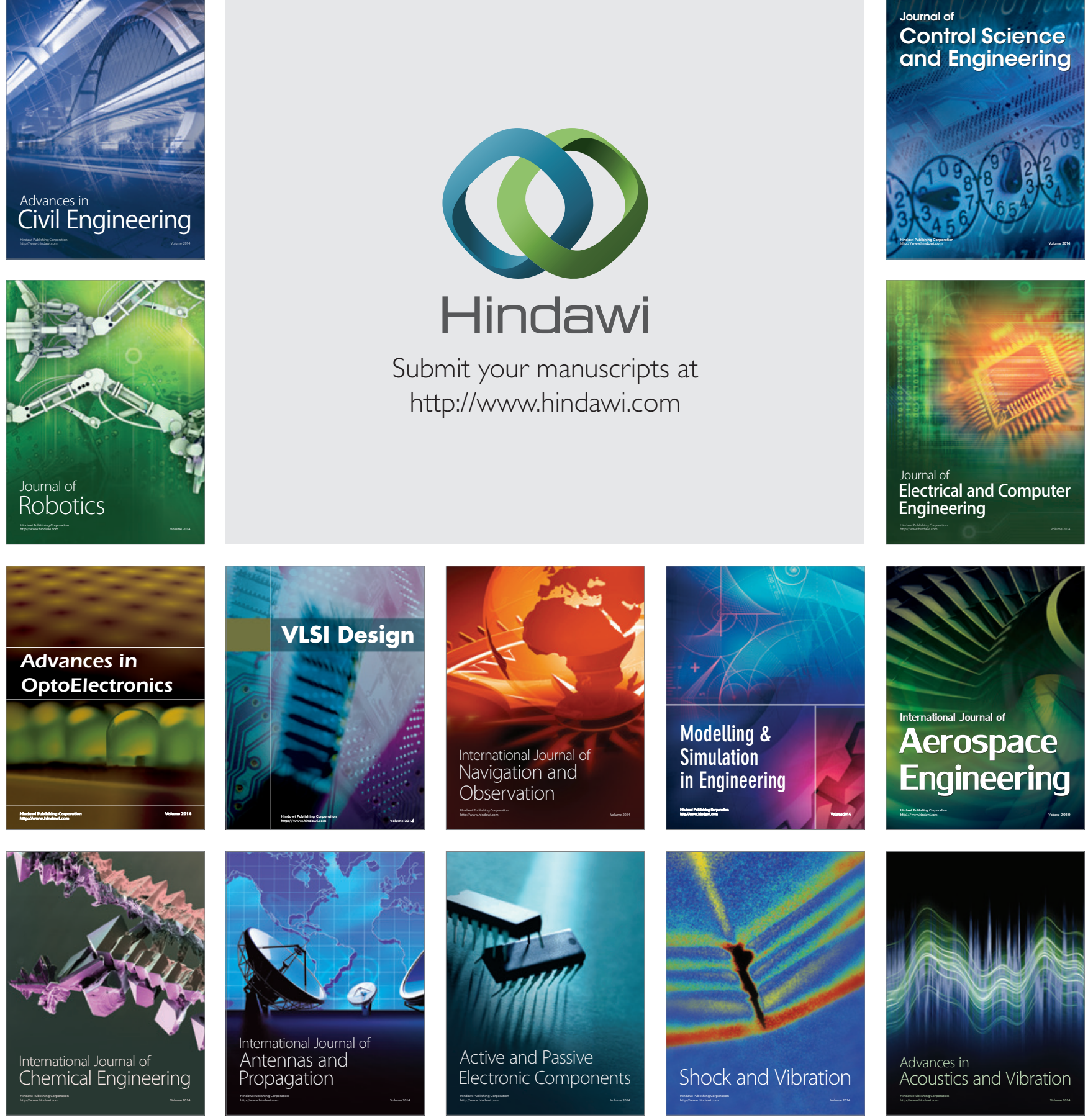